\title{
La transmission de la proposition dansée: lieu de co-construction de la corporéité de l'enseignant et des élèves
}

\section{Dominique Montaud et Chantal Amade-Escot}

Sur la base d'une étude de cas longitudinale d'une durée de six ans, cet article examine la manière dont se construit la corporéité des élèves en danse, en tant qu'objet d'enseignement en Éducation physique et sportive (EPS).. Nous déclinons ici le concept de corporéité selon une modalité pragmatique, spécifique au monde de la danse: les "états de corps". L'étude s'intéresse à la manière dont un professeur ayant une certaine expérience, mais aucune dans l'enseignement de la danse, mobilise au fil des ans, ses états de corps dans le but de transmettre une proposition dansée à ses élèves. Cette proposition dansée relève d'une tâche prototypique en danse, mise en cuvre dans toutes les séances observées. Les résultats montrent qu'au fil du temps l'intention $d u$ mouvement dansé est co-construite entre les élèves et l'enseignant et que ce dernier crée des conditions de plus en plus favorables à la réalisation d'expériences sensibles pour les élèves, contribuant ainsi à enrichir leurs "états de corps dansant».

\section{Introduction}

Dans son analyse philosophique de la danse en tant que pratique artistique, Pouillaude (2009) montre qu'entre l'idéalité du vocabulaire et la performance individuelle intervient l'entre-deux des façons de faire et façons d'être du danseur, donnant à la danse une qualité particulière. C'est cet entre-deux que Godard (2002) qualifie de corporéité. Contrairement à la danse classique, certains chorégraphes en danse contemporaine, à l'instar de Mathilde Monnier, mobilisent «une écriture par matière» (Pouillaude, 2006, p. 159)) dans laquelle seuls les paramètres généraux de l'identité du geste sont écrits comme: les thèmes kinesthésiques, la rythmique, sans pour autant en fixer la forme. Il s'agit chaque soir pour le danseur-interprète de re-éprouver l'expérience sensible de cet entre-deux plus que de réitérer la forme à l'identique du mouvement. Si de nombreux auteurs ont étudié la corporéité en danse, peu se sont intéressés aux liens entre la corporéité du chorégraphe - objet mais aussi support de transmission - et celle de ses danseurs interprètes comme l'étude didactique de Dufor et Amade-Escot 
(2002) le fait à propos de la transmission par Pina Bausch du rôle de «'élue» dans le «sacre du printemps» à la danseuse Kyomi.

L'inscription de cette étude dans le milieu scolaire est motivée par le contexte particulier de la politique de développement de parcours artistiques et culturels à l'école initiée par le Ministère de l'éducation nationale (MEN). L'enjeu de cette mesure est de permettre à tous les jeunes d'accéder à la culture artistique grâce à la réalisation d'expériences sensibles (MEN, 2013). C'est à travers le prisme de la transmission de «la proposition dansée» (Harbonnier-Topin, 2012) que cet article étudie la manière dont la corporéité d'un enseignant d'EPS et celle de ses élèves s'influencent mutuellement. La recherche, menée durant six années selon une approche ethnographique, étudie les interactions entre l'enseignant et ses élèves dans quatre classes de sixième à propos de savoirs spécifiques relatifs à la corporéité en danse. Elle rend compte de l'évolution de la mise à l'étude de ces savoirs en tant qu'objets d'enseignement.

Enseigner est l'occasion de «donner vie à des pratiques de savoir, en référence à des pratiques socio-historiquement cristallisées en activité» (Schubauer-Leoni, Leutenegger, Ligozat \& Fluckiger, 2007, p. 53). Ainsi, décrire et comprendre l'agir des acteurs de l'action conjointe en didactique nécessite de travailler à la croisée d'une étude épistémologique et didactique. C'est donc aux fondements de l'acte de danser et du concept de corporéité que nous nous intéressons ci-après.

\section{Fondements épistémologiques de la danse: de la corporéité aux "états de corps"}

\section{Quand y a-t-il danse artistique?}

La danse comme tout art ne préexiste pas à l'acte de danser. Aussi, tout comme Goodman (1988) renonce à définir "ce qu'est l'art» pour y substituer la question "quand y a-t-il art?», nous mettons au jour, en nous appuyant sur une revue de littérature en philosophie de l'art, les conditions nécessaires permettant de reconnaître «qu'est ce qui fait danse?». Cette question centrale pour analyser, comprendre, penser les modalités de l'enseignement artistique en milieu scolaire, taraude les didactiques artistiques (Mili \& Rickenmann, 2005). Danser est une activité strictement humaine, intentionnelle. Même si leurs mouvements ont des propriétés esthétiques, les lapins ne peuvent danser car ils n'en ont pas l'intention souligne Pouivet (2010). Dans l'acte artistique le danseur donne à voir une suite de métamorphoses infinies par lesquelles le corps ne cesse de se transformer (Valéry, 1960). Le geste produit par le danseur est juste, «aisthétique» ajoute Despres (2003). Cette auteure le caractérise comme «un mode de production signifiante lié à un processus par lequel la sensation du mouvement mis en ouvre coïncide exactement avec le mouvement de la sensation» (ibid, p. 322). Quelles qu'en soient les gestualités, l'acte artistique de danser a une visée communicative. Cet acte intentionnel est «un processus de remaniement struc- 
turel, contextuel et sémantique grâce auxquels il acquiert une signifiance autre» (Félix, 2011, p. 129). L'usage du corps, spontané ou appris qu'en fait le danseur, relève d'une technique du corps au sens de Mauss (1950), saturée par des savoirs culturellement reconnus quant au produire ce qui fait danse. C'est à la question de la technique du danseur-interprète que nous consacrons la section suivante.

\section{La danse artistique comme technique du corps?}

Plusieurs usages du terme technique coexistent en danse. Un premier désigne une technique de danse donnée, en tant que système gestuel articulé, fait d'entités discrètes, nommables et transmissibles: la technique classique en tant que codification du ballet occidental, mais aussi la technique Graham ou Limon, du nom du chorégraphe et du fondateur de l'école qui transmet cette technique. En temps qu'idéalités, ce système articulé permet de reconnaitre le même dans le différent: une arabesque, un tour en dedans, mais aussi un "contract-release» de Martha Graham, un "curve» de Merce Cunningham; et cela quel que soit le niveau d'exécution du danseur. Un second usage du terme technique permet de porter un jugement sur la compétence du danseur à incorporer ces entités gestuelles: par exemple «tel danseur a une bonne ou mauvaise technique». Au-delà des entités nommables, des niveaux d'exécution, Pouillaude (2009) considère qu'il y a des façons de faire et surtout des façons d'être. Ainsi selon cet auteur, «Pirouette» ou "Grand jeté» s'ancrent sur un fond postural et dynamique. Dans ce troisième usage, le fond postural, c'est-à-dire l'activité anticipatrice (i.e. gestion des appuis, maintien de l'équilibre par les muscles "gravitaires», placement du bassin, projection du sternum en avant, port de tête, etc...) conditionne le mouvement qui va suivre: la façon de faire. Le fond dynamique (i.e. impulsion du mouvement, accentuation du phrasé, etc...) joue sur la façon d'être. Le croisement du fond postural et dynamique donne ainsi au mouvement une signature singulière indépendamment de tout vocabulaire et participe d'une certaine forme de corporéité (Godard, 2002). Ainsi, danser pour le danseur-interprète repose fondamentalement sur un arrière-fond postural et dynamique: l'entre-deux déjà évoqué, tel que le définissent Pouillaude (2009) et Godard (2002). Nous caractérisons la spécificité de la corporéité en danse dans les lignes qui suivent.

\section{De quelle corporéité se préoccupe-t-on en danse?}

Merleau-Ponty (1945) s'est attaché à démontrer l'inadéquation et le caractère fallacieux du concept de «corps» tel qu'entendu dans la culture occidentale, c'està-dire celui d'un corps anatomique, constitué de parties matérielles composant l'organisme, siège des fonctions physiologiques. Le concept phénoménologique de "chair" proposé par cet auteur vise à interroger la façon dont chaque être «est au monde et incarne ce corps». Dans la même veine, Bernard (2001) substitue aux concepts de "corps» et de "chair», celui de "corporéité», qu'il définit comme une «entité affective, subjective et symbolique» induisant pour chaque être un 
type singulier de rapport au monde. Ici, la dimension sociale et historique de la construction du corps est minorée au profit de l'expérience sensible qui redéfinit indéfiniment ce rapport au monde. Bernard (2001) conçoit la corporéité en danse comme un spectre sensoriel et énergétique d'intensités hétérogènes et aléatoires. Pour le spécifier il revisite les propositions de Merleau-Ponty (1965) relatives au fonctionnement chiasmatique de nos sensations. Il y majore la part "fictionnelle» que génère le sentir de chacun dans la corporéité. C'est en s'appuyant sur les dimensions phénoménologiques du concept de corporéité développées par Bernard (2001), que les théoriciens contemporains ainsi que les formateurs en danse en spécifient le sens dans ce champ en proposant le concept, selon eux plus pragmatique, «d'état de corps» qui désigne ce qui préside à une qualité particulière de danse (Guisgand, 2012; Louppe, 1997; Pouillaude, 2009).

\section{Les "états de corps": une approche pragmatiste de la corporéité en danse}

Dans la dynamique d'infinies métamorphoses qui caractérise la danse, les auteurs pointent un dénominateur commun, sorte d'invariant auquel l'interprète peut faire appel lorsqu'il danse: «noyau eidétique» pour Pouillaude (2009), «état de corps» pour Guisgand (2012). Ce dernier concept, de nature descriptive, vise à fixer "un antidote à la désagrégation permanente» du mouvement dansé. La perspective pragmatique développée par Guisgand considère les «états de corps» comme «l'ensemble des tensions et des intentions qui s'accumulent intérieurement et vibrent extérieurement et à partir duquel le spectateur peut reconstituer une généalogie des intensités présidant à l'élaboration d'une forme corporelle ou d'un mouvement» (ibid, p. 33). Cet auteur accorde le primat à l'intention, celle qui induit les modes tensionnels conditionnant le mouvement. L'intention ne relève pas tant de l'état d'esprit dans lequel le danseur se trouve au moment de sa performance mais des conditions de l'apparition de son mouvement dansé. En nous appuyant sur Baxendall (2000) nous considérons que les institutions traversées par le danseur, les expériences sensibles éprouvées, ainsi que les techniques incorporées participent de l'intention. Aussi choisirons-nous de parler de l'intention du mouvement plutôt que de l'intention du danseur. L'originalité du concept d'états de corps est d'entremêler dans un même terme, la dimension poïétique ${ }^{1}$ du corps dansant (celui du danseur) avec la dimension aesthésique $^{2}$ du corps contemplé par le spectateur. Pour ce dernier, l'état de corps du danseur ne relève pas d'une qualité objective mais d'une interprétation prédiquée: «il est la résultante perçue de la confrontation de deux corporéités: celle, dansante, de l'interprète et celle, sentante, du spectateur» (Guisgand, 2012, p. 33). L'état de corps est à la fois interprétation par le spectateur de «l'effet de surface» que produit «une manière d'être» du danseur, et appréciation métaphorique de ce qui est perçu, incorporé, senti par le spectateur. L'état de corps du spectateur renseigne aussi sur le degré de porosité de sa sensibilité. L'expérience sensible, que le spectateur réalise est liée à sa capacité à percevoir et à apprécier 
les dynamiques internes sous jacentes aux états de corps du danseur. Ce concept, profondément ancré dans la théorie de l'empathie décrite par Berthoz (2004) fonctionne sur l'idée d'une contamination kinesthésique du spectateur par le danseur davantage que sur celle, décrite par Faure (2000), de «l'intercorporéité». Accéder pleinement à la réception d'un mouvement dansé, nécessite pour le spectateur de l'avoir lui-même pratiqué car le mouvement nous touche par où nous le côtoyons. Ainsi la pratique de la danse en tant que danseur, facilite sa réception et sa compréhension en tant que spectateur (Guisgand, 2010). Cet éclairage théorique légitime la demande institutionnelle de confronter les élèves aux rôles de danseur, chorégraphe, spectateur, dans un même cours de danse en EPS (MEN, 2008). Dans le cadre particulier de la transmission d'une proposition dansée ces rôles sont tenus simultanément. Les états de corps du danseur, qu'il soit professeur ou élève(s), agissent en classe sur ceux du spectateur (professeur ou élèves). Aussi ancrer la recherche dont rend compte ce chapitre dans le cadre des Études de l'action conjointe en didactique (EACD) semble particulièrement pertinent et inexploré à notre connaissance.

\section{Une recherche inscrite dans le cadre théorique de l'action conjointe en didactique}

Selon cette approche, tout acte social est distribué entre plusieurs acteurs, l'activité de l'un requérant l'activité de tous les autres pour pouvoir se déployer. Ainsi, agir dans le monde social, c'est s'ajuster à autrui et agir conjointement (Mead, 2006). La question des sémioses y est centrale, les actions de chacun des acteurs faisant signes, en tant que signaux d'action, pour les autres. Les pratiques d'enseignement-apprentissage mettent en jeu à la fois la maîtrise de jeux de langage spécifiques, un processus sémiotique particulier et l'émission d'inférences conjointes à partir de l'environnement (Sensevy, 2010). En considérant que l'individu prend comme stimulus à la racine de ses actes le comportement d'autrui en autant de signaux d'action, l'analyse de l'action conjointe contribue à revisiter les liens entre verbal et non verbal dans les processus d'enseignement-apprentissage. L'énonciation didactique, les prosodies dont le professeur fait usage, le regard dans les interactions, la proxémie constituent autant de signes à interpréter pour les acteurs de l'action conjointe (Sensevy, 2011). Parce que les «états de corps» sont un rapport au monde qui se donne à voir, ils peuvent être également perçus par autrui comme signes potentiels, le professeur et l'élève étant respectivement sémaphore l'un pour l'autre. Les signes ont une valence perlocutoire, le professeur parle et agit pour faire agir l'élève à propos de savoirs. Ces derniers sont au cœur des transactions didactiques lors de l'action conjointe.

La modélisation de l'action conjointe en didactique à partir d'un système de descripteurs: contrat et milieu didactique; mésogenèse, topogenèse et chronogenèse; définition, régulation, dévolution et institutionnalisation (pour 
des définitions de ces concepts, voir Mili \& Leutenegger dans l'introduction de ce numéro thématique) rend possible l'analyse ascendante de la transposition didactique (Schubauer-Leoni \& Leutenegger, 2005), permettant d'accéder aux savoirs réellement mis à l'étude, dans cet article ceux relatifs à la proposition dansée.

\section{Problématique et questions de recherche}

Comme indiqué précédemment, la transformation des états de corps des élèves constitue l'un des enjeux de savoir de la transmission de la proposition dansée 3 . Savoir est entendu ici comme une puissance d'agir. Il s'agit, dans cette étude, d'identifier comment les états de corps d'un enseignant-danseur font signe aux élèves danseur-interprètes et comment en retour le professeur prend en compte dans son enseignement ce qu'il perçoit des états de corps des élèves.

Une première difficulté, relève du double statut des acteurs de l'action conjointe: l'enseignant en performant sa chorégraphie donne à voir des états de corps à ses élèves, mais il est aussi spectateur des leurs. Symétriquement, l'élève est à la fois spectateur de l'enseignant-danseur, mais aussi producteur responsable de ses états de corps. Aussi, lors de la transmission d'une proposition dansée, les états de corps du professeur et des élèves relèvent d'un double registre, celui poïétique du corps dansé et celui aesthésique du corps contemplé: comment dénouer ce subtil entrelacs? Pour résoudre ce problème nous envisageons de rendre compte de façon relationnelle de ce que font et disent l'enseignant et les élèves à propos des états de corps qu'ils produisent et contemplent.

Mais par ailleurs, les états de corps de ces deux acteurs résonnent avec ceux du chercheur-spectateur. En effet, s'ils relèvent d'éléments tangibles similaires, l'état de corps dansant n'est pas entièrement superposable à l'état de corps contemplé. Aussi, adopter une posture d'estrangement au sens de Ginsburg (1998), nécessite pour le chercheur d'établir des critères suffisamment objectifs pour caractériser les états de corps de l'enseignant et des élèves. Quelles solutions adopter d'un point de vue méthodologique?

\section{Protocole méthodologique}

Pour étudier, dans l'action conjointe, comment les états de corps d'un enseignant et ceux d'élèves-danseurs de classes de sixième participent des transactions didactiques en danse, nous avons construit un système d'observations multidimensionnel croisant trois échelles temporelles et deux granularités d'analyse. Nous avons suivi un enseignant chevronné (que nous appellerons Pierre) pendant 6 ans. Cet enseignant est novice dans l'enseignement de la danse au moment où débute la recherche, puis progressivement formé, lors de trois stages de formation continue, effectués lors des quatre premières années de l'étude longitudinale. L'échelle macro-didactique analyse les cycles de danse observés (structure du cycle, tâches proposées) afin de repérer, dans les séances, des tâches récurrentes 
et suffisamment voisines pour être candidates à la comparaison micro-didactique (Montaud, 2014). Parmi ces tâches, nous retenons pour cet article, celles relevant de la «transmission d'une proposition dansée» créée par l'enseignant. Ce type de tâches est prototypique en enseignement de la danse (Harbonnier-Topin, 2012). C'est aussi la seule tâche dans laquelle Pierre se met en jeu corporellement et pour laquelle ses états de corps sont à la fois objet mais aussi supports des transactions didactiques. Dans les propositions dansées retenues, les états de corps de Pierre et de ses élèves font l'objet d'une analyse micro-didactique qui s'inscrit dans la lignée de l'approche clinique expérimentale du didactique ordinaire (Leutenegger, 2009). La retranscription des données recueillies met en relation les entretiens (ante-cycle et post séance), les interactions verbales et la description des actions de Pierre et des élèves à partir des séances filmées. Pour retranscrire le mouvement dansé nous mobilisons certains termes techniques de la notation en danse (par exemple pour une position: "petite seconde, bras en première»). Les corpus ainsi élaborés constituent des systèmes de traces permettant de procéder à une "description épaisse» (au sens de Geertz, 1986) des transactions didactiques à partir du croisement d'une part, des différentes formes sémiotiques mobilisées par les acteurs dans l'action conjointe et d'autre part, des entretiens avec Pierre. Ces traces permettent des interprétations en profondeur (Mary, 1998) relatives à l'évolution des milieux et contrats didactiques, à l'avancée des savoirs en jeu et aux topos des acteurs. D'un point de vue anthropologique ce protocole a pour intérêt d'articuler, comme le défend Sensevy, des interprétations robustes et dans «le langage du modèle», et dans "la sémantique familière de l'action ${ }^{4}$ (Sensevy, 2007).

\section{Tableau 1: Exemple de mise en relation des traces recueillies dans l'action} conjointe pour documenter les états de corps en l'an1

\begin{tabular}{|c|c|}
\hline $\begin{array}{l}\text { Interactions verbales à partir des enregistre- } \\
\text { ments audio }\end{array}$ & $\begin{array}{l}\text { Transcription du mouvement dansé à partir des } \\
\text { traces vidéographiques }\end{array}$ \\
\hline \multicolumn{2}{|l|}{ Première phrase de la proposition dansée } \\
\hline \multirow[t]{2}{*}{$\begin{array}{l}\mathrm{P}: \text { «On est posé les deux pieds au sol, les bras le long } \\
\text { du corps on écarte doucement les mains on ouvre } \\
\text { les bras on rapproche nos mains on les frotte, on les } \\
\text { frotte, là on les écarte, on les écarte» }\end{array}$} & $\begin{array}{l}\text { P: Petite seconde, bras en bas/ écarte les bras/ } \\
\text { paume contre paume frotte les mains } 3 \mathrm{x} / \text { Écarte } \\
\text { les bras/ les laisse tomber sous leur poids }\end{array}$ \\
\hline & $\begin{array}{l}\text { É: reproduisent à l'identique regard fixé sur le } \\
\text { professeur }\end{array}$ \\
\hline \multicolumn{2}{|l|}{ Deuxième phrase de la proposition dansée } \\
\hline $\begin{array}{l}\text { P: «Là on va faire comme si on s'appuyait sur l'air, } \\
\text { c'est pas mes mains qui bougent c'est mon corps qui } \\
\text { bouge vous voyez mes mains, elles restent fixes, en } \\
\text { avant en arrière, en avant en arrière, deux fois vers } \\
\text { l'arrière vers l'avant» }\end{array}$ & $\begin{array}{l}\text { P: bras légèrement écartés mains en pronation/ } \\
\text { mains fixes/ translation buste vers l'avant initié } \\
\text { par le sternum/ appuis orteils/ réoriente mains } \\
\text { en supination/ translation vers l'arrière par les } \\
\text { vertèbres dorsales, appuis talons }\end{array}$ \\
\hline $\begin{array}{l}\text { É: «c'est comme quand on fait des pompes les mains } \\
\text { bougent pas» }\end{array}$ & $\begin{array}{l}\text { É: Pas d'isolation du buste, ni des mains: balancé } \\
\text { antéro-postérieur du corps, initié par tête/ } 6 \\
\text { élèves: déséquilibre vers l'avant }\end{array}$ \\
\hline
\end{tabular}


Nous recherchons dans les interactions verbales et non verbales retranscrites, des indices pour documenter les états de corps de Pierre et des élèves. En restant au plus près des éléments exposés dans la partie théorique (Guisgand, 2012; Pouillaude, 2009), nous avons retenu plusieurs critères pour le faire: intentions du mouvement du danseur; fond dynamique (i.e. la généalogie des intensités sous-tendant son élaboration, point de déclenchement); fond postural du danseur (i.e. appuis, placement du bassin, projection du sternum, port de tête, regard). Selon les usages méthodologiques de la recherche en didactique nous débutons la section des résultats par l'analyse a priori de la tâche "transmission d'une proposition dansée» afin d'identifier les savoirs en jeu et les éventuelles difficultés pour les élèves à la lumière desquels nous interprétons les actions produites.

\section{Les états de corps: analyse de l'action conjointe entre professeur-danseur et élèves-interprètes}

Nous présenterons ci-après quelques extraits permettant de documenter les états de corps de Pierre et de ses élèves lors de la transmission de la proposition dansée en l'an1 et l'an2 de la recherche. L'an2, comme nous le montrerons, constitue une bascule qualitative dans les monstrations de Pierre. C'est à ce titre que nous nous y intéressons en montrant, brièvement, dans un second temps les tendances d'évolution entre ces deux années et au-delà, au fil de la recherche longitudinale.

\section{Analyse a priori de la tâche: transmission de la proposition dansée}

Cette tâche est prototypique de la manière dont les savoirs du danseur sont mis à l'étude en EPS (Harbonnier-Topin, 2009). Elle est mise en place par Pierre lors de toutes les séances observées. Il s'agit selon les propres termes de l'enseignant d'une tâche visant à «apprendre un module» (entretien post-séance). L'analyse porte sur la tâche générique qui, au-delà des visées d'acquisition de différentes formes de mouvement dansé, du vocabulaire y afférant et des états de corps la sous-tendant, relève aussi de ce qui peut être considéré comme la mise à l'étude d'une œuvre au sens de Meyerson (1948), ici celle produite par le professeur pour être transmise. Pierre, pour tous les cycles observés, procède par accumulation de phrases simples, peu techniques. Il présente aux élèves plusieurs monstrations réitérées d'un court module de danse (1 à 2 minutes), le plus souvent accompagné verbalement. La "proposition dansée» du professeur, son degré de difficulté technique, ses qualités d'amplitude, d'intensité, de rythmicité, de présence par le regard, les états de corps ainsi produits définissent le milieu didactique primitif à partir duquel se déploie l'action conjointe. Au fil des transactions, les monstrations successives et les énoncés contribuent à le préciser. Dans ce "contrat d'imitation" au sens de Sensevy (2011) proposé par Pierre, plusieurs types de savoirs sont en jeu pour s'approprier la proposition dansée: 
- Percevoir les signes pertinents dans la monstration du professeur et les transformer en puissance d'agir;

- Enchaîner les mouvements sans les juxtaposer pour donner à voir des moments de danse;

- Se rendre sensible aux états de corps de l'enseignant comme matériau pour produire les siens.

Une première difficulté réside dans l'instantanéité et la pluralité des actions du professeur et des différentes parties de son corps, rendant ce milieu particulièrement dense. Une deuxième est d'interpréter les signes corporels discursifs dont fait ostentation le professeur lorsqu'il transmet la proposition dansée afin de leur donner du sens pour que les élèves se les approprient. Notons que la linéarité du langage et sa diachronie ne peuvent rendre compte du syncrétisme et du synchronisme du mouvement dansé. "Entendre dire» un mouvement dansé constitue également une difficulté majeure pour celui qui l'entend pour la première fois et qui n'en a pas l'usage. Par ailleurs, dire un mouvement dansé relève d'un jeu de langage au sens de Michaud (1999) que les élèves de sixième ne possèdent pas encore. Pour les programmes officiels de danse en EPS (MEN, 2008), mais également pour les élèves, danser relève d'une reproduction de formes plus que de l'appropriation d'états de corps singuliers à chaque élève-danseur: aussi les états de corps de Pierre, peuvent-ils ne pas faire signe aux danseurs. Une troisième difficulté réside, pour les élèves, dans la possibilité (ou non) d'auto-évaluation de leur performation grâce uniquement aux actions (verbales et non verbales) du professeur. Enfin la quatrième difficulté provient du «contrat d'imitation» qui force un rapport effectif au milieu pour l'élève tentant d'agir adéquatement.

\section{Évolution des états de corps dans l'action conjointe du professeur et des élèves}

Dans cette section nous présentons différents extraits situés à des moments caractéristiques de l'enquête: c'est-à-dire rendant compte des manières dont dans l'action conjointe les états de corps sont mis à l'étude ou servent de support pour Pierre dans la tâche de transmission de «la proposition dansée».

\section{Les états de corps lors de la transmission de la proposition dansée: an1}

Nous retenons un extrait tiré de la séance 2 du cycle mis en place en 2007. Composée de cinq phrases très statiques, la proposition dansée est une juxtaposition d'actions quotidiennes en référence au vécu des élèves: "on écarte les mains»; "on ouvre les bras». Dans la phrase 2, une translation antéro-postérieure du buste constitue la seule difficulté. Elle est impulsée par le sternum, entraînant un déplacement du poids du corps sur l'avant des pieds, puis un passage en appui-talon. Les bras écartés en diagonale basse constituent un appui fixe pour l'avancée du sternum. Dans la plupart de ses mouvements Pierre reste en seconde parallèle pour maintenir son équilibre. Seul un changement vient rompre la linéarité du flux et de la rythmicité des mouvements, lors d'un cercle 
de bras impulsé vivement par la main. Pierre, face aux élèves (ce qui n’aide pas à la lecture, ni à l'exécution par les élèves du mouvement du fait de sa position en miroir), concentré dans sa tâche de monstration, reste davantage dans le contrôle de son action et de son accompagnement verbal que dans l'écoute de ses sensations produites par ses mouvements: en conséquence il ne produit pas de moments de danse.

L'analyse des registres lexicaux mobilisés dans l'accompagnement verbal, pour définir le milieu ou réguler les actions des élèves, pointe l'utilisation prépondérante de verbes d'action (16) en pertinence avec l'action soutenue: «on rapproche nos mains», "on descend» (verbatim). La fonctionnalité de ces verbes est de proposer une "gestalt» (au sens de forme signifiante immédiatement disponible) favorisant l'auto-organisation de l'expérience corporelle et permettant à l'élève d'agir sans passer par une analyse cognitive spatiale et temporelle coûteuse comme l'a mis en évidence Harbonnier-Topin (2009). Peu de verbes (trois) indiquent - et dans ce cas, de façon imprécise - la posture initiale, support au mouvement: "on est posé sur les pieds». Aucune indication ne précise les parties du corps déclenchant les mouvements. Lorsque Pierre initie sa translation vers l'avant par le sternum, il en tait l'information aux élèves, ayant des difficultés à situer le point de déclenchement: "c'est le corps qui bouge». Une seule indication précise l'intensité consentie au mouvement: "on ouvre doucement les mains". Pour signifier que l'air pourrait servir d'appui fictif pour les mains, lors de la translation, Pierre a recours à une analogie: "on va faire comme si on s'appuyait sur l'air». Grâce à cette condensation de sens (Tribalat, 1995) Pierre poursuit sa monstration sans en interrompre le cours, tout en indiquant l'intention de son mouvement.

Nous retenons qu'en cet an 1 d'observation, le soutien verbal de Pierre repose sur une sémantique familière, peu technique. Les verbes d'action énoncés privilégient la dimension cinématique aux dépens d'indications sur le "fond postural» du mouvement. Par ailleurs, sur le plan perlocutoire, on note un déficit d'indications relatives à son «fond dynamique». Nous concluons, puisque Pierre tait dans son soutien verbal toute information relative à ses états de corps, qu'il dévolue involontairement aux élèves la charge d'interpréter les dimensions dynamiques du mouvement dansé qu'il produit et donne à voir dans sa monstration. Dans l'action conjointe, sur le plan mésogénétique, et contrairement aux modalités du soutien verbal en usage dans les cours en danse contemporaine (Faure, 2000), cet enseignant ne réussit pas complètement à indiquer aux élèves les traits pertinents du mouvement dont ils doivent se saisir. L'analyse du film montre que la plupart des élèves, en position de danseurs, les yeux rivés sur Pierre, tentent d'imiter les éléments de surface du mouvement: écarter les bras, dessiner un cercle avec le bras, etc... Ils manifestent cependant des difficultés dans la reproduction de certains éléments du fond postural (au sens de Godard, 2002 et Pouillaude, 2009) de Pierre. Ils adoptent une position, pieds serrés, peu stable. Dans la deuxième phrase, les élèves transforment le mouvement initial de translation du 
buste de Pierre en un balancé antéro-postérieur de tout le corps, impulsé par la tête. Les régulations verbales de Pierre ("mes mains restent fixes") restent improductives car ce dernier ne décrypte pas, dans l'action des élèves, leur difficulté à initier le déclenchement de la translation par le sternum. L'analogie poétique, «s'appuyer sur l'air», ne fait pas non plus sens pour les élèves. L'un d'entre eux la reformule d'ailleurs en référence à une expérience motrice connue: "c'est comme quand on fait des pompes, les mains bougent pas", ce qui renforce notre interprétation. Pris dans un contrat didactique de reproduction au plus près de la cinématique des mouvements, les élèves gardent en permanence le regard fixé sur Pierre, ce qui les empêche d'éprouver le mouvement et de se l'approprier.

Il ressort de cette analyse que si les élèves reproduisent les éléments cinématiques et le déclenchement dynamique de mouvements simples, empruntés à leur motricité quotidienne, le milieu didactique proposé par Pierre ne réussit pas à créer des conditions de réception de sa proposition dansée. Les élèves réinterprètent certains éléments relatifs au fond postural (appuis) et au fond dynamique (avancée du sternum). Ils transforment l'intention du mouvement ("on fait des pompes») en restant dans un registre de surface. Pierre fait preuve pourtant d'une certaine sensibilité dans ses régulations relativement aux éléments de surface des mouvements (à titre d'exemple, en indiquant aux élèves de partir dans telle ou telle direction). Ses régulations restent cependant infructueuses lorsqu'elles concernent les états de corps des élèves, ce qui amène Pierre à rabattre ses intentions didactiques: "la logique de ce mouvement il est lent, j'aurais aimé que ... le saut devienne rapide et les mouvements de bras fort, qu'ily ait vraiment la différence entre lent et rapide, c'est difficile de faire passer ça...» (entretien post-séance, an1)

\section{Les états de corps lors de la transmission de la proposition dansée: an2}

Dans ce deuxième extrait de séance, observée en 2009, la proposition dansée de Pierre est composée de trois phrases peu techniques à partir de verbes d'actions quotidiennes («attraper, jeter, regarder»). Pierre les enchaîne, contribuant à donner de la fluidité à ces mouvements. Seule l'onde de bras dans la phrase 2, déclenchée par un mouvement d'épaule présente une difficulté. Sa position, dos aux élèves est prototypique de la transmission en danse. Elle libère son port de tête car son regard n'est alors plus fixé sur les élèves (comme c'était le cas en l'an1). Par la fluidité de sa gestuelle, la justesse du regard, les modulations d'intensité et de rythmicité accordées au mouvement, Pierre produit des «moments de danse», malgré encore quelques maladresses dans ses mouvements.

L'évolution la plus tangible concerne les dimensions mésogénétiques de l'accompagnement verbal de la monstration. Pierre, utilise toujours un langage familier. Le nombre d'occurrences de verbes d'action porteurs d'informations cinématiques augmente significativement (55). Pierre débute sa monstration, de façon ludique: «vous avez tous une poche? Regardez ce qu'il y a... une balle!». Tel un fil conducteur, il déroule cette "métaphore filée» (Commandé, 2011) à propos d'une balle imaginaire logée dans la poche et avec laquelle il joue, tout 
au long de la monstration. Cette figure de style indique aux élèves l'intention de sa proposition dansée. La métaphore filée est prétexte à de nombreuses comparaisons: "on la bloque comme un gardien»; "on la lance». D'un point de vue mésogénétique ces analogies transposent le sens initial du mouvement ("fermer les bras en s'accroupissant, lever le bras") dans le champ sémantique de la balle. La formulation métaphorique favorise ainsi un meilleur contrôle du mouvement et indique, implicitement la généalogie de sa dynamique (Cadopi, 1999): les verbes «lancer», "bloquer» sont porteurs d'une intensité très forte et d'une vitesse rapide en début d'action, puis décroissante. L'usage d'interjections: «et hop", de prosodies: "on baaaaaaaascule la balle» émaillent l'accompagnement verbal de Pierre et participent de la mise en lumière de la dynamique du mouvement. Ce procédé est délibéré. Il a pour fonction de condenser les informations: «et $d u$ coup ça suit derrière il n'y a pas besoin de dire vite lent fort» (entretien post-séance). Pierre, lors de cet an2, insiste aussi sur le placement du regard.

En cet an2 d'observation, la métaphore filée, les analogies à propos de la balle fictive, ainsi que la sonorisation du soutien verbal qui caractérise la manière dont Pierre transmet la proposition dansée, agissent en synergie et valorisent des indications relatives à la dynamique du mouvement. Les indications sémiotiques de Pierre, entremêlant description cinématique et dynamique, signalent une évolution qualitative des transactions didactiques relatives aux états de corps entre Pierre et ses élèves. Soulignons ici, qu'elles sont aussi prototypiques des usages d'enseignement de la danse contemporaine (Cadopi, 1999).

Les élèves adhèrent tous au jeu de la métaphore filée, certains la prolongent et poursuivent en aparté le jeu fictif. Ainsi, nous observons qu'une fille se met à jongler avec la balle imaginaire, ou encore qu'un garçon dribble avec elle. Au fil des réitérations de la monstration (quatre), les élèves lâchent Pierre des yeux et s'abandonnent à ses indications verbales. Si certains gestes, d'un point de vue cinématique, ne sont pas analogues à ceux de Pierre, comme par exemple l'onde de bras, on observe cependant que la dynamique de leur mouvement est juste au sens de l'appropriation de l'état de corps visé par Pierre. Les régulations verbales de Pierre restent souvent inefficaces car imprécises quant au point de déclenchement de l'onde: «il y a un petit mouvement de corps». Il tait notamment l'impulsion par l'épaule (cf. analyse a priori). Malgré les évolutions observées, ce constat conforte notre interprétation selon laquelle Pierre n'a d'autre choix que de rabattre son intention didactique. S'il privilégie, lors de l'an2, l'aspect dynamique du mouvement, il en tait encore la composante technique qui permettrait pourtant aux élèves de l'éprouver. Il reste que les images vidéo, et l'analyse que nous en faisons, indiquent que dans le décours de l'action conjointe les élèves produisent cependant des mouvements aisthétiques (Despres, 2003). Nous nous autorisons à dire que nous sommes en présence de moments de danse rendus possibles par une mise en jeu corporelle relevant de ce que Félix (2011) nomme: «état de corps dansant», caractérisés par des "durées intérieures» et des «modalités de présence totale» du sujet à l'action qu'il vit. 
Pour tenter de comprendre pourquoi les élèves dès l'an 2 produisent des moments de danse que nous retrouvons les années suivantes, alors qu'en l'an1, ils ne reproduisaient que des formes approximatives, nous mettons en discussion dans la section suivante les rapports entre états de corps du professeur et états de corps des élèves.

Vers des "états de corps dansant»

Précisons tout d'abord que les élèves des classes successivement observées ne présentent pas de traits typiques particuliers, ce qui nous amène à porter la focale sur l'évolution des états de corps de Pierre et leur résonance sur ceux des élèves. Dès l'an2, nous pointons une évolution, voire une rupture, qui va perdurer: la juxtaposition des formes qui caractérise la proposition dansée à l'an1 se transforme en une dynamique de métamorphoses, décrite ci-après. Tout d'abord Pierre, en l'an2, joue sur les modulations toniques et les modulations temporelles du mouvement, selon un curseur allant de l'accélération à l'arrêt, d'un flux libre à un flux bloqué. De même, la qualité et la précision du placement de son regard concourent à donner une dimension singulière à sa proposition dansée: une façon d'être, une façon de faire que nous appelons pour l'instant «états de corps».

Cette tendance se confirme dans le soutien verbal de la monstration. Par le truchement de la métaphore filée à propos de la balle, Pierre crée les conditions d'un milieu didactique favorable à l'identification par les élèves de l'intention de sa proposition dansée. "La fabrique du sens» qui en découle organise ainsi leur expérience du geste et donne le sens (direction) aux sens, selon l'expression de Godard (1994). Cette intention permet aux élèves de passer d'une simple reproduction de forme (comme dans l'an1) et que Paillard (1971) nomme «morphocinèse» à une production de sens, c'est-à-dire à des «sémiocinèses» selon Serre (1984) qui les définit comme des formes motrices présidant aux relations entre l'homme et son environnement social à des fins de communication symbolique. Notons, sans pour autant pouvoir le développer dans le cadre de cet article, que cette tendance s'accentue encore lors de l'an 4 d'observation. Pierre, de plus en plus à l'aise avec cette figure de style, est en mesure de dévoluer aux élèves l'avancée de la métaphore filée: "et maintenant on fait quoi avec cette balle?» (verbatim, an4). Par cette sollicitation, il engage les élèves à participer plus personnellement à l'élaboration de l'intention de la proposition dansée. Cette dernière est ainsi co-construite, selon un processus de sémiogenèse collective dont les prémices, apparues en l'an2, se développent les années suivantes. Les nombreuses analogies (métaphore, comparaison, etc.) précisent au fil des ans une partie du fond dynamique du mouvement dansé proposé par Pierre, contribuant ainsi à densifier le milieu didactique et à soutenir la chronogenèse. Dès l'an2, Pierre propose des images adaptées aux élèves, leur permettant de travailler dans un registre proprioceptif (Cadopi, 1999). Les élèves s'approprient alors un mouvement nouveau à partir de significations anciennes ayant des 
enracinements, corporels, sensoriels, émotionnels, conceptuels. Les métaphores sont en effet de "puissants médiateurs sémiotiques permettant d'appréhender l'inconnu, et de structurer le mode d'engagement des acteurs dans des activités nouvelles» (Durand et al., 2013, p. 57). Dans l'enseignement de la danse, nous défendons l'idée que ces métaphores viennent spécifier le milieu didactique en marquant ses dimensions poḯtiques et aesthésiques, ce qui rompt avec les usages habituels de l'enseignement des activités physiques et sportives en EPS. Par ailleurs, et ce dès l'an2 Pierre, nous l'avons vu, insiste sur le regard ce qui contribue à définir une certaine façon d'être pour les élèves. La mise en jeu de ces types de régulations suppose l'acquisition d'un langage nouveau, seul à même à maintenir vif le rapport à la pratique de référence artistique.

Il reste que les éléments relatifs au fond postural évoluent peu. Les indications concernant les appuis restent ténues. Nous avons aussi pointé des stagnations. Par exemple, Pierre reste réticent à préciser les points qui initient le mouvement, soit par ignorance de leur importance, soit par difficulté à décrire ce qu'il réalise pourtant avec une précision accrue au fil des années. C'est pourquoi les élèves simplifient les mouvements les plus complexes, ce qui ne les empêche nullement de produire des états de corps.

En résumé, il ressort de cette étude longitudinale que l'accompagnement verbal de Pierre se précise au fil des ans, donnant des indications de plus en plus précises quant à l'intention du mouvement, à son fond postural et dynamique. Monstration, soutien verbal, modulations de la voix, convergent et renseignent complémentairement les élèves sur les états de corps de Pierre. Chacun d'eux, chargé de ses propres expériences sensori-motrices et émotionnelles construites dans un contexte culturel particulier, s'approprie de façon singulière les signes qu'il saisit lui permettant d'entrer dans un rôle de danseur-interprète. Dès l'an2, les élèves donnent à voir un certain type d'états de corps et le spectateur (incluant le chercheur-spectateur) peut capter des signes relatifs à l'intention du mouvement, aux appuis, à la relation à la pesanteur, au déclenchement du mouvement et à sa dynamique. Les états de corps des élèves et de Pierre dans les moments de danse, nous amènent à penser que, au fil des quatre observations sur les six années de l'étude longitudinale, Pierre et les élèves sont progressivement en mesure de produire "des états de corps dansant» de moins en moins fugaces au fil des ans. Nous revenons dans la discussion qui suit sur le concept d'état de corps dansant et sur les conditions de sa construction dans l'action conjointe.

\section{Discussion conclusive: La corporéité objet de L'apprentissage et moyen de sa transmission en danse en EPS}

L'étude que nous avons menée met au jour toute l'importance de l'intention dans la co-construction des «états de corps dansant» lors de la transmission de 
la proposition dansée: «La dynamique ou les qualités de la danse proviennent des intentions du danseur traduite en mouvement» (Guisgand, 2012, p. 33). Nous avons montré que ces états ne préexistent pas à l'intention ni au procès expérientiel qui les fondent (Félix, 2011). Les états de corps dansant émanent d'une corporéité d'action teintée par l'intention, mais aussi par la sensation éprouvée du mouvement (Guisgand, 2012). Nous avons bien conscience qu'une analyse fonctionnelle du mouvement nous aurait permis de mieux analyser l'arrière fond postural des danseurs, le pré-mouvement qui, selon Godard (1995), participe de l'état de tension du corps et de la qualité du mouvement. Au-delà de cette limite, notre étude souligne toutefois que l'état de corps des élèves n'est, en effet, pas premier: il ne préside pas à l'élaboration des formes. La dimension poïétique de leur mouvement résonne sur sa dimension aesthésique, en retour cette dernière affecte la dimension poïétique. Comme le souligne Bernard (2001) les états de corps du danseur résonnent sur la manière dont le spectateur «fictionne l'instant» et ressent la vibration extérieure de l'auto-affectation du danseur. Une des hypothèses de Guisgand (2010) est que la danse nous arrive par le corps et qu'ainsi sa pratique amplifie sa perception. Selon lui, la danse nous affecterait par où on la côtoie! Nous considérons que les expériences sensibles réalisées par les élèves ou Pierre en tant que spectateur(s) et danseur(s) jouent sur leur réception du mouvement. Les états de corps dansant (des élèves et de Pierre) sont donc nourris à la fois par les expériences poïétiques et aesthésiques réalisées dans d'autres contextes, moteurs, émotionnels, culturels mais aussi par les expériences relatives aux états de corps contemplé (du professeur ou des élèves). Autant de contextes d'action susceptibles de participer à la réorganisation et à la revisite des expériences d'états de corps: «L'état de corps contemplé relève d'une corporéité perceptive fut-elle nourrie des souvenirs d'action du spectateur» (Guisgand, 2012, p. 33).

Si l'on veut rendre à la danse en EPS sa dimension culturelle, comme le stipulent les recommandations institutionnelles (MEN, 2008) et ne pas la limiter à une activité de production de formes, un des enjeux - à nos yeux fondamental, pour un réel accès à la culture artistique en milieu scolaire - est de créer les conditions pour que les élèves réalisent des expériences d'états de corps, pour en «dégager de la matière sensible» (au sens de Gaillard, 2005) à partir de leur éprouvé. Savoir saisir cette matière dans l'ostension du professeur et en faire l'expérience singulière devient dès lors une puissance d'agir (un savoir) pour l'élève danseur-interprète. C'est en en préservant toute la dimension phénoménale et expérientielle que l'on peut, selon nous, échapper à «l'ethnocentrisme didactique en danse» décrié par Félix (2011), celui qui contribue à réifier les savoirs en objet d'enseignement. Créer les conditions pour faire réaliser des expériences à propos des états de corps, tout comme produire un jeu de langage pour les dire, s'apprend et se construit (Michaud, 1999). Dans le cas de Pierre, cet apprentissage relève d'une sémiose partagée avec ses élèves. Les expériences réalisées chaque année en contexte d'enseignement et en stage de formation 
continue, agissent sur l'action didactique de Pierre et contribuent à remanier et à densifier ses compétences dans l'enseignement de la danse, ce qui a des conséquences sur les acquisitions des élèves.

Dans cet article nous avons tenté d'éviter le piège d'une distinction entre états de corps du danseur et états de corps du spectateur, même si l'exercice d'écriture nous a obligé à en rendre compte de façon diachronique. Les danseurs (professeur et élèves) sont dans une position particulière dans laquelle les différentes impressions sensorielles, celles éprouvées par leur mouvement en train de se faire, et celles produites par la contemplation du mouvement de l'autre, sont rassemblées dans une unité de sensations que nous rapprochons du «sentir et se mouvoir» de Strauss (1989). C'est à ce titre que la tâche de transmission d'une proposition dansée nous intéressait. En pensant l'activité du professeur et des élèves liées intrinsèquement, le modèle théorique de l'action conjointe nous a permis d'envisager les transactions à propos des états de corps sur le mode de deux subjectivités en dialogue. C'est pourquoi nous avons traité la corporéité non pas seulement comme un média de l'action didactique conjointe mais en soulignant combien la spécificité des savoirs qui la concerne, notamment en danse, rendent encore plus cruciale la nécessité de démêler ce qui, dans la corporéité, est au service des sémioses collectives, de ce qui est l'enjeu même du savoir à enseigner et à apprendre. C'est pour rendre compte de cette distinction que nous avons convoqué le concept d'état de corps. Le concept d'état de corps - dansant ou contemplé - nous a permis de décliner la notion de corporéité en danse telle qu'envisagée par Bernard (2001), tout en la formalisant à la suite de Guisgand (2012) et Pouillaude (2009) comme un rapport au monde de chaque être selon une approche pragmatique qu'il reste cependant à développer et à enrichir.

\section{Notes}

1 La dimension poïétique est liée aux procédures de production de l'objet artistique.

2 La dimension aesthésique est liée aux processus de réception de cet objet.

3 Ces enjeux de savoir sont développés dans la section 5.1. Analyse a priori de la tâche: transmission de la proposition dansée

4 Définie comme: «l'activité telle qu'elle peut apparaître aux familiers de l'action telle qu'ils peuvent se la décrire entre eux» (Sensevy, 2007, p. 42).

\section{Ré férences}

Baxandall, M. (2000). Formes de l'intention. Nîmes: Jacqueline Chambon.

Bernard, M. (2001). De la création chorégraphique. Paris: Centre national de la danse.

Bernard, M. (2002). De la corporéité fictionnaire. Revue internationale de philosophie, 422, 523-534.

Berthoz, A. (2004). L'empathie. Paris: Odile Jacob.

Cadopi, M. (1999, mai). Nature et rôle des consignes pour l'apprentissage moteur en danse. Communication présentée au colloque international "La danse une culture en mouvement», Strasbourg.

Commandé, E. (2011). La danse artistique une activité motrice de symbolisation. Consulté le 12 Février 2013 dans http://www.passeursdedanse.fr/pdf/la_danse_artistique_une_activite _motrice_de_symbolisation.pdf 
Despres, A. (2003). Travail des sensations dans la pratique de la danse contemporaine, Logique du geste aisthétique. Lille: ANRT.

Dufor, F. \& Amade-Escot C. (2002). La transmission chorégraphique chez Pina Bausch - Analyse didactique d'une répétition. In C. Fleischle-Braun (Éd.), Actes du colloque: Tanz zwischen den Kulturen: Tanz als Medium der interkulturellen Bildung und Identitätsentwicklung; Symposium am Institut für Sportwissenschaft der Universität Stuttgart (1.-3.10.2001) (pp. 96-104). Butzbach: Afra-Verl.

Durand, M., Goudeaux, A., Horci, Z., Salini, D., Danielan, J. \& Frobert, L. (2013). Expérience, mimesis et apprentissage. In L. Albarello, J. M. Barbier, E. Bourgeois, \& M. Durand (Éds.), Expérience, activité, apprentissage, (pp. 39-59). Paris: PUF.

Faure, S. (2000). Apprendre par corps. Socio-anthropologie des techniques de danse. Paris: La Dispute.

Félix, J. J. (2011). Enseigner l'art de la danse. Bruxelles: De Boeck.

Gaillard, J. (2005). Expérience sensorielle et apprentissage. Approche psycho-phénoménologique. Paris: L'Harmattan, Collection Santé, société, culture.

Geertz, C. (1986). Savoir local, savoir global: les lieux du savoir. Paris: PUF, Sociologie d'aujourd'hui.

Godard, H. (1994). Le souffle le lien. Marsyas, 232, 27-31.

Godard, H. (2002). Le geste et sa perception. In M. Michel \& I. Ginot (Éds.). La danse au $20^{e}$ siècle (pp. 236-241). Paris: Larousse.

Goodman, N. (1988). Quand y a-t-il art? In G. Genette (Éd.), Philosophie analytique et esthétique (pp. 199-210). Paris: Klincksieck.

Ginzburg, C. (1998). Occhiacci legno. Nove riflessioni sulla distanza. Milan: Milan Editore.

Guisgand, P. (2010). Lire le mouvement dansé. Revue STAPS, 89, 85-89.

Guisgand, P. (2012). Étudier les états de corps. Spirales, 242, 33-34.

Harbonnier-Topin, N. (2009). Autour de la proposition dansée, regard sur les interactions professeur-élève dans la classe technique de danse contemporaine. Thèse de doctorat de Sciences de l'Éducation. Paris : CNAM.

Harbonnier-Topin, N. (2012). Le temps de la transmission en danse: de la construction des savoirs et des pratiques à l'élaboration de traces chorégraphiques. Montréal: UQAM et CRF-CNAM.

Leutenegger, F. (2009). Le temps d'instruire. Berne: Peter Lang.

Louppe, L. (1997). Poétique de la danse contemporaine. Bruxelles: Contredanse.

Mauss, M. (1950). Sociologie et anthropologie: les techniques du corps (pp. 363-386). Paris: PUF.

Mary, A. (1998). De l'épaisseur de la description à la profondeur de l'interprétation. À propos de «Thick description». Enquête, 6, 57-72.

Mead, G. H. (2006). L'esprit, le soi, et la société. Paris: PUF.

MEN (2008). Programme d'éducation physique et sportive du collège. Bulletin officiel spécial de l'Éducation nationale, numéro 6.

MEN (2013). Les parcours d'éducation artistique et culturelle. Circulaire $\mathrm{n}^{\circ}$ 2013-073 du 3-5-2013.

Merleau-Ponty, M. (1945). Phénoménologie de la perception. Paris: Gallimard.

Merleau-Ponty, M. (1965). Le visible et l'invisible. Paris: Gallimard.

Meyerson, I. (1948). Les fonctions psychologiques et les æeuvres. Paris: Albin Michel.

Michaud, Y. (1999). Critères esthétiques et jugement de goût. Nîmes: Éditions Jacqueline Chambon.

Mili, I. \& Rickenmann, R. (2005). La construction des objets culturels dans l'enseignement artistique et musical. In C. Moro \& R. Rickenman (Éds.), Situation éducative et significations (pp. 165-196). Bruxelles: De Boeck.

Montaud, D. (2014). Le rapport aux cuvres dans l'enseignement de la danse au collège. Analyse didactique de l'évolution de l'épistémologie pratique d'un professeur d'EPS. Thèse de doctorat de Sciences de l'Éducation. Université Toulouse 2 - Jean Jaurès.

Paillard, J. (1971). Les déterminants moteurs de l'organisation de l'espace. Cahiers de psychologie, 4, (4), 261-316. 
Pouillaude, F. (2006). Vouloir l'involontaire et répéter l'irrépétable, pour une approche phénoménologique. In A. Boissière. Approche philosophique du geste dansé. pp.145-160). Presse universitaire du Septentrion.

Pouillaude, F. (2009). Le déseuvrement chorégraphique. Étude sur la notion d'aeuvre en danse. Paris: Vrin.

Pouivet, R. (2010). Danse, ontologie et philosophie de l'âme. In J. Beauquel \& R. Pouivet (Éds.) Philosophie de la danse (pp. 33-44). Rennes: PUR.

Schubauer-Leoni, M. L. \& Leutenegger, F. (2005). Une relecture des phénomènes transpositifs à la lumière de la didactique comparée. Revue Suisse des sciences de l'éducation, 27, (3), 407-429.

Schubauer-Leoni, M.L., Leutenegger, F., Ligozat, F. \& Fluckiger, A. (2007). Un modèle de l'action conjointe professeur-élèves: les phénomènes didactiques qu'il peut/doit traiter. In G.Sensevy \& A. Mercier (Éds.), Agir ensemble. L'action didactique conjointe du professeur et des élèves (pp. 51-91). Rennes: Presses Universitaires.

Sensevy, G. (2007). Des catégories pour décrire et comprendre l'action didactique. In G. Sensevy \& A. Mercier (Éds.), Agir ensemble. L'action didactique conjointe du professeur et des élèves, (pp. 13-49). Rennes: Presses Universitaires.

Sensevy, G. (2010). Esquisse d'une pragmatique didactique. Psychologie de l'interaction, 27-28, 179-223.

Sensevy, G. (2011). Le sens du savoir, Éléments pour une théorie de l'action conjointe en didactique. Bruxelles: De Boeck.

Serre, J. C. (1984). La danse parmi les autres formes de motricité. La recherche en danse, 3, 135-156.

Strauss, E. (1989). Du sens des sens: contribution à l'étude des fondements de la psychologie (G. Thinès \& J. P. Legrand, trads.) Grenoble: Éditions Jérôme Million. (Original publié en 1935).

Tribalat, T. (1995). La danse discipline artistique. Hyper, 192, 18-26.

Valéry, P. (1960). L'Âme et la Danse. Paris: Gallimard.

Mots-clés: Danse, corporéité, états de corps, action conjointe en didactique, éducation physique, proposition dansée

\section{Die Vermittlung von Choreographien: ein Ort der gemeinsamen Konstruktion von Körperlichkeit durch Lehrer und Schüler}

\section{Zusammenfassung:}

Auf der Grundlage einer longitudinalen Fallstudie, die sich über sechs Jahre erstreckte, untersucht dieser Artikel, auf welche Weise Schüler ein körperliches Bewusstsein im Tanzen als Unterrichtsgegenstand des Faches Sport/Leibeserziehung entwickeln und erlernen. Das Konzept der "Körperlichkeit» wird hier in einer pragmatischen Sichtweise als "Körperhaltung» («états de corps») verstanden und durchbuchstabiert, wie es für den Bereich des Tanzens typisch ist. Die qualitative Studie untersucht, auf welche Art und Weise ein Lehrer, der zwar gewisse Erfahrungen im Sportunterricht, nicht aber im Unterrichten von Tanzen hat, im Laufe der Zeit seine eigenen Körperhaltungen entwickelt und 
einsetzt, um seinen Schülern eine bestimmte Choreographie beizubringen. Die Vermittlung von Choreographien zum Tanzen stellt eine prototypische Lehrund Lernaufgabe dar, und die untersuchten Lehrer implementieren diese Art der Aufgabe in allen Stunden, die sie unterrichten. Die Ergebnisse zeigen, dass Lehrer und Schüler die ästhetische Absicht der Tanzbewegungen über die Jahre gemeinsam konstruieren. Sie zeigen auch, dass der Lehrer im Laufe der Zeit mehr und mehr in der Lage ist, entsprechende Lernbedingungen zu erstellen, die den Schülern reichhaltige, sensible und einzigartige Erfahrungen bieten, welche es ihnen ihrerseits erlaubt, ihre «tanzende Körperhaltung/ihre körperliche Verfassung» zu verbessern und zu verfeinern.

Schlagworte: Tanz, Körperlichkeit («corporéité»), Körperhaltung/körperliche Verfassung («états de corps»), gemeinsames didaktisches Handeln, Sportunterricht, Choreographie

\section{La trasmissione della coreografia di danza: luogo di co-costruzione della corporeità dell'insegnante e degli allievi}

\section{Riassunto}

Sulla base di uno studio longitudinale di caso durato sei anni, questo articolo esamina il modo in cui si costruisce la corporeità degli allievi nella danza, quale oggetto d'insegnamento in educazione fisica e sportiva. Decliniamo qui il concetto di corporeità secondo una modalità pragmatica, specifica al mondo della danza: gli «stati del corpo». Lo studio si interessa al modo in cui un docente che ha una certa esperienza, ma nessuna nell'ambito della danza, mobilizza negli anni i suoi «stati del corpo" con l'obiettivo di trasmettere una coreografia di danza ai suoi allievi. La trasmissione della coreografia si rivela un'attività d'insegnamento tipica, attuata in tutte le lezioni osservate. I risultati mostrano che con il tempo l'intenzione del movimento danzato è co-costruita fra gli allievi e l'insegnante e che quest'ultimo crea condizioni sempre più favorevoli alla realizzazione di esperienze sensibili per gli allievi, contribuendo così a arricchire i loro «stati del corpo danzante».

Parole chiave: Danza, corporeità, stati del corpo, azione congiunta in didattica, educazione fisica, coreografia danzata 


\title{
The transmission of dance choreography: a place where teacher's and students' corporeity is co-constructed
}

\begin{abstract}
Based on a six years longitudinal case study, this paper examines how students construct a corporeal knowing in dance as a teaching object within physical education (PE). The concept of "corporeity» is studied through the notion of «states of embodiment» («état de corps») with respect to a pragmatist approach specific to the world of dance. This longitudinal study is carried out with an experienced teacher, but having no experience in PE. It focuses on the personal «states of embodiment» he unfolds when transmitting an intended choreography to his students. The transmission of choreography in dance is a prototypic teaching task the teacher implements in all the lessons we observed. Results show that the intented dancing movement is co-constructed by the teacher and the students; moreover, the former, over the years, generates more and more learning conditions offering students with rich, sensitive and singular experiences that contribute to foster their "states of dance embodiment» («états de corps dansant»).
\end{abstract}

Key-words: Dance, corporeity, states of embodiment, didactic joint action, physical education, choreography 